\title{
Forgotten ureteral double-J stents and related complications: a real-world experience
}

\author{
Sachin Patil ${ }^{*}$, Kshitij Raghuvanshi, Devendra Kumar Jain and Abid Raval
}

\begin{abstract}
Background: Double-J (DJ) stents are among the basic and commonly used tools in urology. The DJ stent generally needs to be replaced or removed within 6 weeks to 6 months to avoid complications like encrustations, stone formation, fractures and blockades of stents. However, in many cases the stent is forgotten. In this retrospective study, we report our experience in the management of forgotten stents and steps taken by us in preventing DJ stent-related morbidity.
\end{abstract}

Results: Of the total of 30 patients, $80 \%(n=24)$ underwent previous procedures in other hospitals and the remaining 20\% $(n=6)$ were from our hospital. The mean age of the patients was 56.66 years. The mean duration of the indwelling stent in situ was 13.83 months. The indications of indwelling stents included ureteroscopic lithotripsy, extracorporeal shock wave lithotripsy and percutaneous nephrolithotomy. Presenting complaints for which patients visited hospital were flank pain $(n=10 ; 33.33 \%)$, dysuria $(n=9 ; 30 \%)$, hematuria $(n=3,10 \%)$ and fever $(n=2 ; 6.66 \%)$.

Conclusion: Forgotten DJ stents can be a source of severe morbidity. Patients' and relatives' educational level and counseling before and after the procedure may play a significant role in reducing stent-related complications.

Keywords: Complications, Forgotten double-J stent, Real-world experience

\section{Background}

Double-J (DJ) stents are among the basic and commonly used tools in urology in many procedures since its first introduction in 1967 by Zimskind et al. [1]. These stents keep the ureter patent and ensure resolution of any edema and allow for any injury. Hence, it is considered as an effective method in postoperative management in patients with ureteric calculi, ureteric stricture, retroperitoneal tumors or fibrosis, ureteropelvic junction obstruction or in any iatrogenic ureteric injury.

In patients with obstructive uropathy secondary to urinary calculi, DJ stent is generally the first choice of treatment. However, there are several morbidities associated with the use of DJ stents. Zimskind et al. also reported

\footnotetext{
*Correspondence: drsachin.uro@gmail.com

Department of Urology, Bharati Vidyapeeth Medical College and Hospital, Pune 411043, India
}

complications associated with the use of DJ stents [1]. The DJ stent has been known to have various short-term and long-term complications. Short-term complications include infection, hematuria, pain and stent syndrome. Long-term retention of stents can lead to encrustations, stone formation, fractures and blockades of stents, hydronephrosis and at times loss of renal function. The incidence of encrustation over DJ stent increases with the duration that the stent remains indwelling [2].

It has been reported that the stent encrustation rates are $9.2 \%, 47.5 \%$ and $76.3 \%$ if stent remains indwelling for 6 weeks, $6-12$ weeks and more than 12 weeks, respectively [3]. The DJ stent generally needs to be replaced or removed within 6 weeks to 6 months [36]. There are several reports available on the forgotten DJ stent [7-9]. Several procedures are described in the literature for the removal of these stents. The type of procedure depends upon the various factors like extent 
and location of encrustation, whether stent is broken, etc. It is important that all patients with stent placement should be counseled for the long-term complications of indwelling stents and the importance of their removal or replacement.

Forgotten ureteral stents, especially those longer than one year, are heavily encrusted and may need auxiliary procedures like shock wave lithotripsy (SWL), ureteroscopy (URS) and percutaneous nephrolithotomy (PCNL) alone or in combination for complete treatment $[7,10]$. In this paper, we report our experience in the management of forgotten stents and steps taken by us to preventing DJ stent-related morbidity.

\section{Methods}

This was a retrospective study conducted at the Department of Urology, Bharati Hospital, Pune, over a period of 24 months (May 2016 to May 2018). A total of 30 patient's data were collected from medical records, who met the eligibility criteria of forgotten DJ stent ( $>6$ months), and factors like duration of DJ stent indwelling, presenting complaints, and type of previous procedure were noted. Additionally, current procedures performed for the removal of DJ stent and any associated complication were noted. The patients included in this study were those referred from peripheral hospitals as well as previously operated at our institute. All the patients were evaluated with the medical history, socioeconomic status and literacy.

Each patient underwent ultrasonography kidneyureter-bladder (KUB), X-ray KUB, urine analysis and serum creatinine. Non-contrast computed tomography (CT) was performed when indicated (mainly for radiolucent calculi and in complex cases like fractured or broken stent). Sterile urine was ensured before intervention. The plan of treatment was decided on the basis of investigations. Institutional ethics committee waived ethical approval considering retrospective nature of the study.

Over the last few years, we have set a protocol in our department about detailed counseling for the patients with DJ stents. Our hospital database also maintains records of all the patients with DJ stent placement, which include name, age, sex, details of diagnosis, type of procedure, date of surgery, due date of stent removal and contact details. We also take the patient's or relative's signature on discharge card making sure that they are actively involved in necessary follow-ups. In case a patient does not turn up for the stent removal or replacement, reminder call is given. Every effort is made to help patients to follow up on time.
Table 1 Indications of indwelling stents

\begin{tabular}{ll}
\hline Indications & $\begin{array}{l}\text { Number } \\
\text { of cases }\end{array}$ \\
\hline Ureteroscopic lithotripsy & 14 \\
Percutaneous nephrolithotomy & 8 \\
Open pyeloplasty & 3 \\
Extracorporeal shock wave lithotripsy & 2 \\
Laparoscopic pyeloplasty & 2 \\
Ureteric reimplantation & 1 \\
\hline
\end{tabular}

Table 2 Presenting symptoms

\begin{tabular}{ll}
\hline Complications & $\begin{array}{l}\text { Number of cases } \\
\text { (range of symptoms) }\end{array}$ \\
\hline Dysuria & 24 (6 months to 15 years) \\
Storage lower urinary tract symptoms & 16 (6 months to 15 years) \\
Hematuria & 12 (8 months to 11 years) \\
Flank pain & 9 (9 months to 15 years) \\
Recurrent UTI & $8(11$ months to 15 years) \\
\hline
\end{tabular}

\section{Results}

A total of 30 patients were enrolled in this study, of which $80 \% \quad(n=24)$ underwent previous procedures (for which DJ stent was inserted) in other hospitals and the remaining $20 \%(n=6)$ were from our hospital. In all cases, polyurethane was used. The mean age of the patients was 56.66 years, and the age ranged from 23 to 66 years. Of the 30 participants, 19 (63.33\%) were males and $11(36.67 \%)$ were females. The mean duration of the indwelling stent in situ was 13.83 months, and the duration ranged from 6 months to 15 years. Two patients had education above higher secondary level, 12 patients had education below higher secondary level, and 16 patients were illiterate. All hailed from rural India with the poor socioeconomic background.

Twenty-four patients forgot about their stent, and in 6 patients, there was history of inadequate counseling by urologist. The indications of indwelling stents are shown in Table 1 . Most common indications for stenting were URS (46.67\%) and PCNL (26.67\%). A total of 28 patients had encrustations, and two patients had fractured stent.

Table 2 summarizes chief complaints for which patients visited hospital (for forgotten DJ stent). Presenting complaints were dysuria $(n=24 ; 80 \%)$, storage lower urinary tract symptoms $(n=16 ; 53.33 \%)$, hematuria $(n=12$, $40 \%)$, flank pain $(n=9 ; 30 \%)$ and recurrent urinary tract infection $(n=8 ; 26.67 \%)$.

Some patients required multimodality approach when needed with few patients requiring more than 2 procedures for removal of indwelling stents (Table 3). In 21 (70\%) patients, URS was required. PCNL, cystoscopy and 
Table 3 Procedures performed for removal of $D J$ stent

\begin{tabular}{ll}
\hline Indications & $\begin{array}{l}\text { Number of cases (range } \\
\text { of duration of procedure) }\end{array}$ \\
\hline Ureteroscopy & 21 (10 months to 11 years) \\
Percutaneous nephrolithotomy & 11 (11 months to 9 years) \\
Cystoscopy and D J removal & 4 (6 to 15 months) \\
Mechanical cystolithotripsy & 3 (8 to 19 months) \\
Open procedure & 2 (7 to 15 years) \\
\hline
\end{tabular}

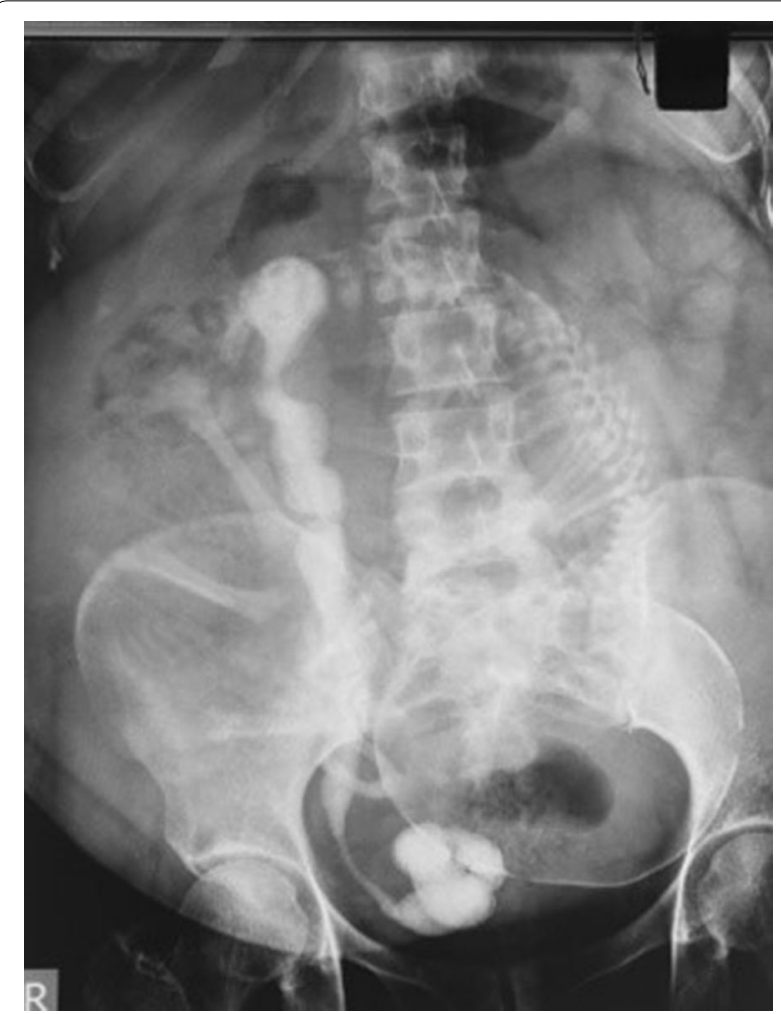

Fig. 1 X-ray KUB scan showing the forgotten stent

DJ stent removal, mechanical cystolithotripsy (CLT) and open procedure for stent removal were required in 11 (36.67\%), 4 (13.33\%), $4(13.33 \%)$ and $2(6.66 \%)$ patients, respectively. One of the pregnant patients who was evaluated in rural area with X-ray KUB was referred to us for management of forgotten DJ stent (Fig. 1). She underwent CLT followed by open procedure for complete stent removal (Fig. 2). This pregnant woman came to us in last trimester and had uneventful normal delivery. The surgery was performed four months after delivery. The whole length of ureter was not incised and incision was taken only at places with stone burden and then by slowly attaining gentle milking we could retrieve the stent. One

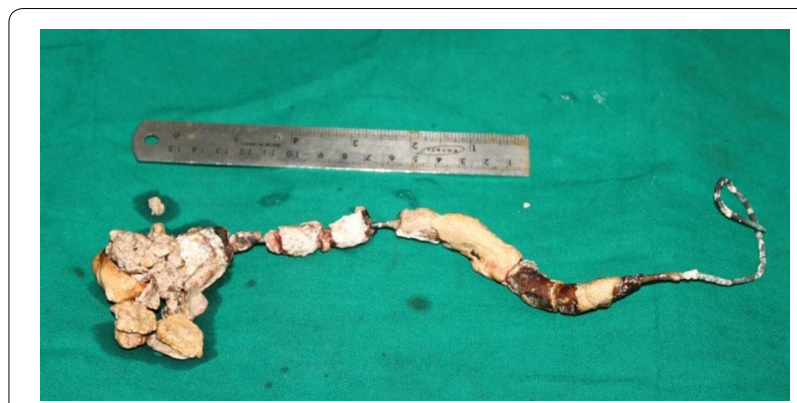

Fig. 2 The removed stent from the patient

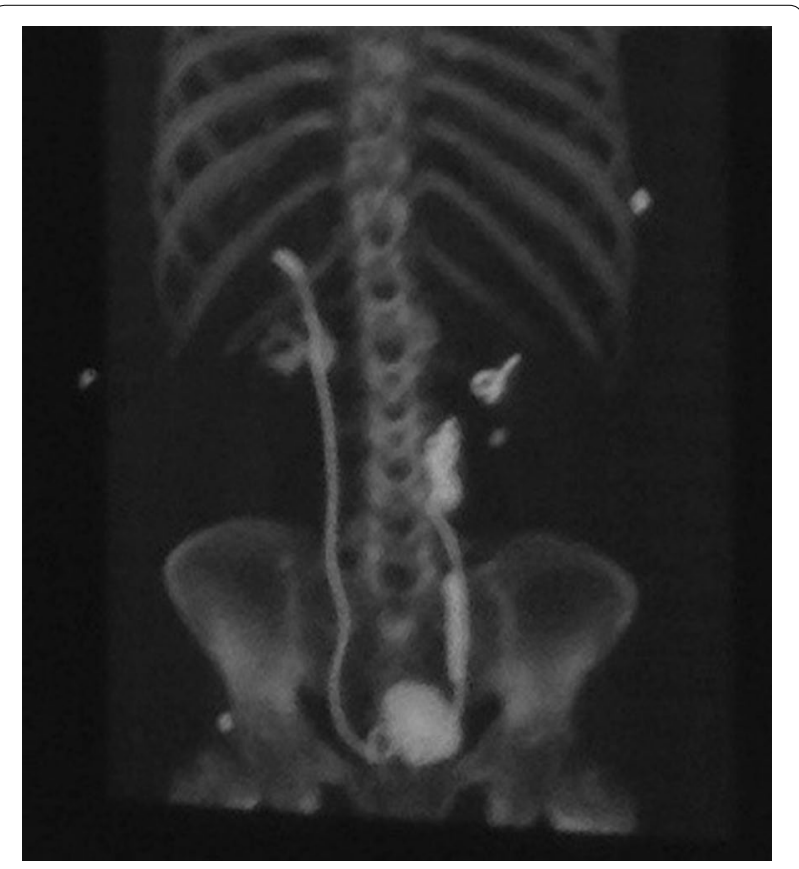

Fig. 3 Fracture present in the stent

patient had fractured upper end of stent on both sides (Fig. 3). She required CLT, open ureterolithotomy and PCNL for complete removal of stent on the left side and cystoscopy with DJ stent removal and PCNL on the right side. Several complications were noted during or after forgotten stent removal like fever (10\%), sepsis (3.34\%), hematuria requiring transfusion (3.34\%) and stent fragmentation $(6.61 \%)$.

\section{Discussion}

Since its introduction in 1967 by Zimskind et al., DJ stent is commonly used in various urological procedures [1]. DJ stents are like double edged sword, if it is kept for long duration or forgotten causing significant morbidity to patient. 
We studied socioeconomic status of patient. We found that most of patients presenting with forgotten DJ stent were from poor socioeconomic background and having low education status. Most of our patients were from rural background, and they were reluctant to travel to tertiary care center in view of poor transportation facility and cost of transportation involved. Singh et al. also reported poor compliance of patients as the main cause for forgotten DJ stent [11]. Improper and hurried counseling by physician also contributed to this problem to certain extent.

Presentation of forgotten DJ stent may vary. We found dysuria $(80 \%)$ and storage lower urinary tract symptoms (53.33\%) as most common presenting symptoms. In a study by Damiano et al., flank pain $(25.3 \%)$ and storage lower urinary tract symptoms $(18.8 \%)$ were most common symptoms [12].

The incidence of complications related to stent increases with the duration of the stent; hence, it is important that it should be removed or replaced on time [3-6]. In our study, stent encrustation, stent breakage and recurrent urinary tract infections were common complications.

In a study by Nawaz et al. [13], the common complications reported were stent encrustation (10.5\%), stent migration (3.5\%) and stent breakage (4.5\%); similarly, in another study, stent encrustation (24.5\%), stent migration $(9.5 \%)$ and stent breakage (1.3\%) were reported as common complications [12]. In another case report, bilateral staghorn calculus was developed due to forgotten DJ stent in a patient with ileal conduit diversion 6 years back [14]. Similarly, Puri et al. reported a large calculus encrusted over a forgotten DJ stent in an ileal conduit patient with urinary diversion 8 years earlier [15].

Treatment of forgotten DJ stent was planned according to extent and severity of encrustation and whether stent was broken. In our study, most patients were managed by endourological approach with few patients requiring open surgery. Multimodality treatment was required in most patients. Similar results were reflected in several other studies as well $[16,17]$. URS and PCNL were most commonly performed procedures for forgotten DJ stent removal in our study with 2 patients requiring open surgery. Several studies describe management of forgotten DJ stent with endourological approach $[7,18]$. We required open procedure in two patients because of severity of encrustation.

We are now concentrating more on patient as well as relative counseling and their active involvement in follow-up. We are taking patient and relative's signature on discharge cards after counseling, and we are showing them post-surgery X-ray KUB explaining them the presence of stent. When patient is lost to follow-up, we communicate with them via telephone or mail. This has helped us in significantly bringing down incidence of forgotten DJ stent.

\section{Conclusion}

Forgotten DJ stent is still a common problem in developing world, and it also brings lot of morbidity and financial burden to patient. This also increases strain on resources and infrastructure which is already limited in developing countries. In most of patients, endourological procedure is required for management of such cases with few requiring open surgery. Proper education and counseling of patients and relatives before and after procedure and maintaining stent register may help in reducing incidence of forgotten DJ stent.

\section{Abbreviations}

CT: computed tomography; CTL: cystolithotripsy; DJ: double J; KUB: kidneyureter-bladder; PCNL: percutaneous nephrolithotomy; SWL: shock wave lithotripsy; URS: ureteroscopy.

\section{Acknowledgements \\ None.}

\section{Authors' contributions}

SP had full access to all of the data in the study and took responsibility for the integrity of the data and the accuracy of the data analysis. SP, KR, DKJ and AR were involved in the study concept and design. SP, KR and AR acquired the data. SP, KR, DKJ and AR analyzed and interpreted the data. SP drafted the manuscript. SP, KR, DKJ and AR were involved in the critical revision of the manuscript for important intellectual content. SP was involved in the statistical analysis. All authors have read and approved the manuscript.

\section{Funding}

This research received no specific grant from any funding agency in the public, commercial, or not-for-profit sectors.

\section{Availability of data and materials}

Not available.

\section{Ethics approval and consent to participate}

Ethical approval was waived from Bharati Vidyapeeth Medical College. Ref: BVDUMC/IEC/115. Date: 18/11/2019. Written informed consent gained.

\section{Consent for publication}

Not Applicable.

\section{Competing interests}

The authors declare that they have no competing interests.

Received: 10 October 2019 Accepted: 15 January 2020

Published online: 12 March 2020

References

1. Zimskind PD, Fetter TR, Wilkerson JL (1967) Clinical use of long-term indwelling silicone rubber ureteral splints inserted cystoscopically. J Urol 97:840-844

2. El-Faqih SR, Shamsuddin AB, Chakrabarti A, Atassi R, Kardar AH, Osman MK et al (1991) Polyurethane internal ureteral stents in treatment of stone patients: morbidity related to indwelling times. J Urol 146:1487-1491

3. Bultitude MF, Tiptaft RC, Glass JM, Dasgupta P (2003) Management of encrusted ureteral stents impacted in upper tract. Urology 62:622-626 
4. Borboroglu PG, Kane CJ (2000) Current management of severely encrusted ureteral stents with a large associated stone burden. J Urol 164:648-650

5. Bukkapatnam R, Seigne J, Helal M (2003) 1-step removal of encrusted retained ureteral stents. J Urol 170:1111-1114

6. Kawahara T, Ito H, Terao H, Yamagishi T, Ogawa T, Uemura H et al (2012) Ureteral stent retrieval using the crochet hook technique in females. PLoS ONE 7:e29292

7. Sohrab A, Aneesh S, Sureka SK, Varun M, Nitesh P, Manoj K et al (2015) Forgotten reminders: an experience with managing 28 forgotten double-J stents and management of related complications. Indian J Surg 77:1165-1171

8. Wani B, Upadhey R, Rathod V, Bhole A (2012) Forgotten long-term indwelling double "J" stent. Saudi J Kidney Dis Transpl 23:1043-1045

9. Bhuiyan ZH, Bhuiyan NI, Khan SA, Tawhid MH, Islam MF (2011) Forgotten urological stent. Mymensingh Med J 20:632-639

10. Patil SM, Magdum PV, Shete JS, Nerli RB, Hiremath MB (2015) Forgotten DJ stent-a source of morbidity: is stent registry a need of the hour. Int J Recent Sci Res 6:2674-2676

11. Singh V, Srinivastava A, Kapoor R, Kumar A (2005) Can the complicated forgotten indwelling ureteric stents be letha? Int Urol Nephrol 37:541-546

12. Damiano R, Olivia A, Esposito C, Desio M, Autorino R, D'Armiento M (2002) Early and late complications of double pigtail ureteral stent. Urol Int 69:136-140
13. Nawaz H, Hussain M, Hashmi A, Hussain Z (1993) Experience with indwelling JJ stents. J Pak Med Assoc 43:147-149

14. Gupta R, Dey RK, Sharma R, Gupta S (2017) Bilateral staghorn calculus with forgotten double J stent in ileal conduit patient-a rare urological challenge. J Clin Diagn Res 11(6):PD09-PD10

15. Puri A, Priyadarshi V, Raizada N, Pal DK (2014) Forgotten DJ stent with a large calculus at its distal end in an ileal conduit diversion. Case Rep Urol 2014:684651

16. Rana AM, Sabooh A (2007) Management strategies and results for severely encrusted retained ureteral stents. J Endourol 21:628-632

17. Monga M, Klein E, Castañeda-Zúñiga WR, Thomas R (1995) The forgotten indwelling ureteral stent: a urological dilemma. J Urol 153:1817-1819

18. Ankur J, Ankur B, Gaurav P, Satyanarayan S (2017) Endourological management of forgotten double J ureteral stents: a single centre study. JOJ Uro Nephron 1:555566

\section{Publisher's Note}

Springer Nature remains neutral with regard to jurisdictional claims in published maps and institutional affiliations.

\section{Submit your manuscript to a SpringerOpen ${ }^{\circ}$ journal and benefit from:}

- Convenient online submission

- Rigorous peer review

- Open access: articles freely available online

- High visibility within the field

- Retaining the copyright to your article

Submit your next manuscript at $\boldsymbol{\nabla}$ springeropen.com 